ing, as she said, tumours per vaginam. The catamenia, when present, were very copious. In April 1844, they stopped, only a few drops passing away. This suppression was followed by lumbar and inguinal pains, colicky pains, and vertigo. In July, pains like labour-pains supervened. A medical man now consulted diagnosed a polypus uteri. Next month, the breasts began to enlarge as well as the abdomen. The movements of a child were felt internally by herself and could be felt externally by the hand. In February 1845, there was recurrence of labour-pains, constipation, and difficult micturition. Eight days subsequently, a kind of membrane (decidua?) came away per vaginam, followed by catamenia for three days. From this time, all fotal movements ceased. The general pains and other symptoms recurred from time to time. The tumour had moved apparently from the median line to the left side. An eminent Parisian accoucheur, now consulted, diagnosed a fibroid tumour in the uterus or ovarian disease. On June 3 , the catamenia recurred, and lasted six days.

On admission, the following were the symptoms observed. By Palpation, etc.: Large abdomen; round hemispheric tumour, more on the right than on the left side; percussion dull anteriorly, clear in the lumbar regions, extending from six centimetres below the xiphoid cartilage to the pubes over a space of thirty centimetres; circumference, seventy-five centimètres; no uterus to be felt, nor fluctuation; external veins not enlarged. When she was placed on her side, the tumour fell to such side, but without pain. There was no souffle. Vaginal Examination: The cervix lay rather to the left side, and hypertrophied, behind the pubes; flattened from before backwards. The os was open, admitting the index finger. Between the vagina and rectum, in the posterior cul-de-sac, a tumour filled the cavity. There was no feeling of a fœetus. M. Jobert diagnosed extrauterine pregnancy and a dead foetus.

The question may be asked, would it be justifiable to make a puncture with an exploratory needle in a case of dubious pregnancy, in the hope of being able to obtain a small quantity of amniotic fluid, and thus diagnosing the correct nature of the case? It seems hazardous to answer this question. Yet it would appear wiser to do so, than to make an exploratory incision and cut down upon the pregnant uterus, as has now been done some three or four times, and always with a fatal result. 'The opinion of Scarpa might be quoted, who dreaded no evil effects from paracentesis abdominis in pregnancy, and who believed the gravid uterus might be safely punctured, and supported his opinion by cases related in the writings of Campa, Brown, Sarquis, and Reiscard. (Cycl. of Prac. Med., iv, p. 385.) In Dr. Hall Davis's case of ovarian pregnancy, the woman was tapped when pregnant, and lived twenty-six days afterwards. So did one of M. Rostan's. In these cases, however, an ordinary trocar was used. A very small opening might not have had the same result; and, by affording a smaller quantity of fluid for examination, might have cleared up the difficulty.

The subject of hydrometra and hcematometra need scarcely detain us. 'The stoppage of the catamenia and fluctuation present would in themselves show that the disease was not of a fibroid character; so, also, in the case of retention of urinc. The introduction of a catheter would remove the difficulty in the diagnosis; and this should always be done in all doubtful cases.

[To be continued.] glllustrations

OF

\section{HOSPITAL PRACTICE:} IIETROPOLITAN AND PROVINCIAL.

\section{ST. GEORGE'S HOSPITAL.}

OBSERVATIONS ON CEREBRAL HEMORRHAGE, FROM AN ANALYSIS OF FORTY FATAL CASES.

By Thomas Jones, Esq., Assistant Resident Medical Officer at the Hospital.

[Continued from page 494 of last volume.]

2. Tremors, Twitchings, Convulsions, Rigidity, and Spasms. At variable periods after the attack of hæmorrhage into the brain, one or more of the above forms of altered motility supervened in 14 cases out of the 40 , i.e. in 35 per cent. In four, two or more of these symptoms were observed in the same patient simultaneously or alternately, but not necessarily affecting the same parts. Thus, in one case there were observed tetanic spasms and tremor affecting the whole body, while there existed at the same time persistent rigidity of both arms. In others, spasms and rigidity were confined to one side, but there were convulsive affections of the whole body.

Tremor. This symptom was noted as having been observed in four of the fourteen cases. It affected the parts paralysed in three, and in the other it was general, while the paralysis was confined to one side. What is its relation to the seat of the clot? In three of the cases the clot was situated in one or more of the ganglia in the neighbourhood of the base; in the other, however, it was situated in the substance of the right middle lobe, but to such an extent, and so near the ventricle, as to completely obliterate it. Thus we find that the clot existed in close contact with the origin of the motor nerve-fibres in all the cases. Again, in three cases, the clot was confined to one side of the brain, in two of which tremors were general, but in the other this symptom was confined to the opposite and paralysed side. In the other case, the clot was situated in the central parts of the pons Varolii, and the tremor was general. In two of the cases, there existed softening of the parts around the clot; in the other two, it is distinctly stated that there was no softening. In the former two, the tremor was slight and occasional; and in the latter, this symptom was more severe and continuous. There seems to be a decided connection between the urgency of this symptom and the duration of life; for in the two cases in which it was severe and general in its extent, the patients lived but few hours; while in the other two in which it was either confined to one side, or to both, but to a slight degree, they lived as many days.

Muscular Twitchings. This symptom was only observed in two cases. It affected the side opposite to the one paralysed in one, and the hand in the other; the muscles of the eyeballs and face alone were mentioned as being paralysed in the latter. The clots in both cases were situated in the meshes of the pia mater over the cerebral hemispheres; in one, there was also some blood in one of the lateral ventricles. No softening was observed around the clot in either of the cases. The patients lived two days after the supervention of this symptom.

Convulsions were noticed in seven cases. The time 
at which this symptom supervened varied from an hour to eighteen days, and after the appearance of this symptom the attack proved fatal in from few hours to seventeen days. In one instance, the patient recovered, but was readmitted for another and fatal attack. Of the six cases in which the parts involved by the convulsions were noticed, they were general in two, in each of which the paralysis was confined to the right side ; in two, the convulsive affections were limited to one side; in one of these the paralysis was on the opposite side, and in the other it was general. In another case, the convulsions affected the muscles of the face, but the paralysis was confined to the left arm and leg. In the other case, the hands were the site of these movements, and the muscles of the face alone the parts paralysed. Thus, there does not seem to be any connection between the seat of the paralysis and the parts affected by these convulsive movements except this, that they are not generally observed on the side paralysed, where this is partial.

What is the relation existing between these convulsive movements and the site of the clot? Of the seven cases, the clot was in contact with the meninges in three, in one of which the crura cerebri and pons, and in another, one of the lateral ventricles, were implicated. In two, the clots were situated in the substance of the hemispheres ; in one, the hæmorrhage was in the corpus striatum, opening into the ventricle; and in the other, the clot was in the centre of the cerebellum near its root. These movements seem generally to occur when the hæmorrhage is either in contact with the membranes on the surface of the brain, or when these extend into the ventricles, or with some of the ganglia at the base. They seldom occur when the clot is situated in the substance. of the cerebral hemispheres, unless there be extensive softening or breaking down of the tissues around, as was noted in the above two cases.

Of six cases, in two the convulsive movements were on the same side of the body as the clot in the brain; in two, the clot was on one side of the brain, whilst the paralysis was general; and in the other two, the clot was on both sides of the brain, and the convulsions were general. It seems to be the prevailing rule that when the lesion is confined to one side of the brain, the convulsions are either general or on the same side of the body-seldom on the opposite side alone. In all the cases except one, there existed softening around a recent clot or old clots and cysts.

Rigidity was observed in five cases, and in all of these this symptom was continuous. It supervened in some cases in few hours; one patient died in two hours after seizure, and another in six hours. It almost always affects the parts paralysed. This symptom was associated in all the cases with a clot in one of the cerebral ganglia; and in all the cases except one, the structure around the clot was softened or broken down.

Tetanic Spasms. This is a phenomenon similar to the latter, but is greater in degree, and more general in extent. These spasms were noted in three cases, each attack being of short duration. They were general in all the cases, and accompanied with rigidity, or rather this symptom was observed in all the cases during the intervals of the spasms. Of all symptoms, this is the most fatal; for of the three cases, one died in few minutes after the supervention of this symptom, and anather, which lived longest, died in six hours. In the former, the parts around the clot were healthy; it was a case of small amount of effusion into both lateral ventricles, issuing from a large dilated vessel in the tænia semicircularis ; in the other two, there was softening around the clot. b. Alterations of Sensibility. Cutaneous ancesthesia and paralysis generally coexisted. In consequence of the frequent existence of coma in these cases, it was difficult, and indeed sometimes impossible, to decide upon the presence or absence of this lesion of sensibility. In all those cases in which this symptom is noted, it always existed in those parts which had lost their power of motility. In few instances, however, where paralysis existed, anæsthesia was absent throughout. The loss of motion is almost always greater than the loss of sensibility. In those cases in which both symptoms coexisted in equal degree, anæsthesia was the one first to pass off. This seems to point out that greater pressure is required upon a nervous centre to cause anæsthesia than to cause paralysis. Of the mucous membranes, that of the eyes was most frequently affected with loss of sensibility. Occasionally the coniunctiva becomes so insensible, that the excito-motory action of the eyelids is completely abolished; so that the finger can be passed over the anterior surface of the eyeball without causing the least movements of the lid, and without the patient evincing the least indication of pain. This I look upon as a very grave symptom, and the patients who present it to a great degree almost always die. Sometimes anæsthesia is confined to one eye, which is generally the one corresponding to the side of the face paralysed.

c. Alterations of the Special Senses. Amaurosis was noted in two cases only; in one of which the lesion in the brain was in the pia mater, and the corresponding cortical substance of both hemispheres. This case tends to show that loss of sight is not always due to pressure upon the tubercula quadrigemina. Very probably it was due, in this instance, to irritation transmitted from the seat of the clot to some parts of the nervous apparatus of vision. In the other case, there was sufficient hæmorrhage, and that in the central ganglia at the base of the brain, which would account for the amaurosis, by the pressure which must have been exerted either upon the tubercula quadrigemina, optic tracts, or the optic commissures. Amaurosis is a symptom more frequently attending cases of softening, abscess, or tumours of the brain, than of hæmorrhage.

Deafness and tinnitus aurium are not mentioned in any of the cases as having occurred.

d. Alterations of Intelligence. In the majority of cases, the intellect was perfectly clear up to the time of the attack; or, if altered, the change was so transient as to escape observation; but in some cases, however, certain alterations were noted: as drowisiness, loss of memory, etc. At the time the hæmorrhage supervened, three conditions of the intelligence were observed.

1. The loss of consciousness was complete. This was observed in the majority of cases; for, out of thirty-eight, it occurred in twenty-six, or 68.4 per cent.

2. Consciousness was but partially abolished. Of the thirty-eight cases, this condition of intelligence occurred in 12, or 31.5 per cent.

3. Lastly, the intelligence was perfectly clear; although, in the cases in which this state was observed, there existed complete hemiplegia.

The chief cause of these differences in the state of the intelligence was the extent of the effusion. The site of the hæmorrhage seems to have influenced the state of consciousness but little. Generally, however, the coma is less deep where the clot is situated in or about the ganglia at the base, than where it occurs near the surface of the hemispheres; for it is interesting to note that, of the thirty-eight cases in which the intelligence was more or less impaired, the principal site of the hæmorrhage was in 
the arachnoid cavity in fourteen; and in all these, with the exception of one, consciousness was completely abolished. Again, of the fourteen cases, there was no paralysis in nine, and of these the hæmorrhage was entirely confined to the arachnoid in four; in five, there existed partial paralysis, but there was lesion in other parts, besides the arachnoid, in all except one.

In most of the cases the coma continued until death-an event which very rapidly supervened. Occasionally, however, the patients lapsed from a state of complete coma into that of partial unconsciousness. In others, again, consciousness was completely re-established; but in these cases the memory was feeble, and some became quite childish in their manner, even shedding tears on being talked to. Most of these cases marked by improvement, were seized by other attacks, of which they died.

As to the relation of loss of consciousness to paralysis, I find that, of the twenty-six cases of complete unconsciousness, there coexisted complete paralysis in eight, partial paralysis in eight, and no paralysis in nine; of the twelve cases of partial loss of consciousness, there coexisted complete paralysis in two, partial paralysis in nine, and no paralysis in one. Affection of the speech has been said to be dependent upon lesion in the antericr lobes. This symptom was noted in three only of the cases under discussion, and in none of them were the anterior lobes implicated. In one case, the arachnoid was the seat of the clot; in another, the middle lobe; and in the third, the corpus striatum.

There does not seem to be a very decided connection between the condition of the pupils and the alterations of intelligence. Of the twenty-nine cases in which the state of the pupils was noted, both were contracted in nine, and dilated in five; and one pupil was dilated in six cases, and one contracted in one. In four cases the pupils were inactive, and in four they were natural. In those cases of altered intelligence in which the pupils were contracted, paralysis existed in 88.8 per cent,; and in those in which the pupils were dilated, paralysis coexisted in 55 per cent.

From the foregoing, I think we may infer that in those cases of deep unconsciousness associated with partial, or with no paralysis, and contraction of both pupils, the clot is most probably in contact with the membranes.

[To be continued.]

\section{Griginal Commurnications.}

\section{RHEUMATIC FEVER : ITS DANGERS AND ITS TREATMENT.}

By John Roperton, Esq., Manchester.

[Read before the Manchester Medical Society, February 3rd, 1364.] I HAVE now for many years taken a deep interest in the treatment of acute rheumatism; and $I$ incline to think (though in this I may be mistaken) that in Manchester we manage the disease more successfully than is done elsewhere. The importance of the subject cannot be questioned, especially when it is considered how large a proportion of all who have had rheumatic fever is found to suffer from some form of heart-affection. Facts in proof of this have long been well known; but none that I have met with are so startling as those lately given to the public in a very able work by Dr. Fleming of Glasgow, entitled Medical Statistics of Life Assurance. In the first place, Dr. Fleming states that, of fifty-seven deaths from disease of heart which occurred in members of the
Scottish Amicable Life Assurance during a period of thirty-five years, as many as one in four had suffered from rheumatism in some form before acceptance. He next gives the results in no fewer than 894 cases of this disease, taken from the works of various authors, which, in Dr. Fleming's opinion, warrant the inference that the heart is affected in at least one-half of the cases of acute and subacute rheumatism; and, lastly, he supplies data illustrative of a most important feature in this disease-its frequent occurrence, and its ill consequences, in early life. Thus, from a table of 379 cases of acute rheumatism at different ages, compiled from the work of Dr. Fuller, we learn that 196 were under the age of twenty-five; and that of these, 110, or a fraction over 56 per cent., had heart-complication.

Dr. Fleming dismisses this portion of his subject in the following words. "Rheumatic fever.may take place at a very early age, as early as the fourth or fifth year, and lay the seeds of irremediable disease of the heart. I have known two such cases where the circumstance was forgotten, and only recalled to mind by the parents, many years afterwards, when serious disease had become evident."

Such being the danger from acute rheumatism, what, we may now inquire, is the treatment best fitted to arrest the disease and so prevent heart: affection? I will state in few words. the remedies I have been in the habit of using for more than thirty years in rheumatic fever; and perhaps the readiest way of doing this will be to give the two following cases. The first is that of a vigorous adult male. After fatigue and unusual exposure to the weather, my patient became feverish, and complained of headache, with acute pain in the right knee. By the evening of the same day, the pain had extended to the ankle, as well as to the other knee; and the following morning the joints of the lower extremities were generally affected, with the addition of pain in the loins and one shoulder. In a few hoprs, nearly all the joints were the seat of acute inflammation. The nature of the case being obvious, a pill, consisting of a grain of opium and two and a half grains of calomel, was administered every three hours till six grains of opium and fifteen of calomel had been taken. The effects were some relief of the pains and a moist skin. I then ordered a purgative mixture, composed of an ounce and a half of Epsom salts and two drachms of the wine of colchicum, in eight ounces of cinnamon water; the dose, a wine-glassful, was repeated every four hours until there was free, not profuse purging. This being accomplished, I prescribed Peruvian bark with quinine, compounded thus : one ounce of powdered cinchona bark, with twenty-four grains of the sulphate of quinine, were shaken up in eight ounces of water, with the addition of a little dilute sulphuric acid to secure the solution of the quinine. Of this a tablespoonful was given every third hour, and also during the night, when the patient was awake. Lemon-juice in water was taken freely as a beverage; and dry cotton-wool, made warm, was wrapped round the joints that were most painful. This course, being steadily maintained-I refer especially to the use of the cinchona and quinine -allayed in a few days the articular inflammation, and speedily effected a cure. Indeed, where this treatment is pursued, I rarely have a patient who is obliged to remain within doors longer than three weeks. Of course, the remedies mentioned have to be varied as to doses, etc., according to the age of the patient and other circumstances. For instance, when the muscles are the chief seat of the disease-a most painful variety of rheumatism-opium may have to be given nightly throughout the illness.

The other case was that of a tall vigorous girl, 